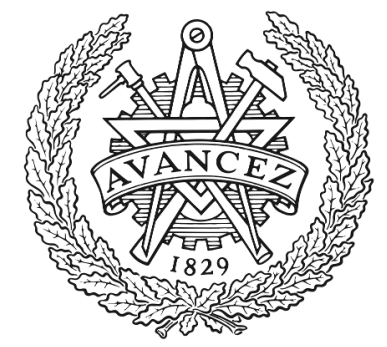

CHALMERS

UNIVERSITY OF TECHNOLOGY

\title{
25 GHz soliton microcombs in high-Q Si3N4 racetrack-shaped microresonators
}

Downloaded from: https://research.chalmers.se, 2023-04-26 15:24 UTC

Citation for the original published paper (version of record):

Ye, Z., Lei, F., Twayana, K. et al (2020). 25 GHz soliton microcombs in high-Q Si3N4 racetrack-shaped microresonators. Conference on Lasers and Electro-Optics, CLEO 2020. http://dx.doi.org/10.1364/CLEO_AT.2020.JTh2F.29

N.B. When citing this work, cite the original published paper. 


\title{
$25 \mathrm{GHz}$ soliton microcombs in high-Q $\mathrm{Si}_{3} \mathrm{~N}_{4}$ racetrack- shaped microresonators
}

\author{
Zhichao Ye*, Fuchuan Lei, Krishna Twayana, Marcello Girardi, Peter A. Andrekson, and Victor Torres- \\ Company \\ Department of Microtechnology and Nanoscience, Chalmers University of Technology, 41296 Gothenburg, Sweden \\ *zhichao@chalmers.se
}

\begin{abstract}
We demonstrate a $25 \mathrm{GHz}$ single-soliton comb on a $\mathrm{Si}_{3} \mathrm{~N}_{4}$ platform fabricated using a novel subtractive method. A long cavity is carefully designed to fit within the e-beam writing field while minimizing coupling to higher-order modes.
\end{abstract}

\section{Introduction}

Dissipative Kerr soliton microcombs have been used in multiple applications, ranging from optical communications to dual-comb spectroscopy [1]. In many of these applications, soliton microcombs operating at repetition rates below $100 \mathrm{GHz}$ are preferred. This represents a formidable challenge for integrated technologies because a larger cavity volume translates into a higher threshold power that must be offset by increasing the quality factor of the microresonator. Indeed, while sub-100 GHz microcombs in $\mathrm{Si}_{3} \mathrm{~N}_{4}$ have been demonstrated before [2], the generation of soliton microcombs at such low repetition rates has been mainly restricted to crystalline and silica microtoroid platforms that offer unrivalled $Q$ values but are challenging to integrate. Only recently, thanks to advances in the socalled Damascene reflow process, with reported mean Qs exceeding 10 million, it has become possible to generate $\mathrm{Si}_{3} \mathrm{~N}_{4}$ microcombs at sub-100 GHz repetition rates [3]. In this fabrication process, the microring resonators are defined by deep UV stepper lithography, and the $\mathrm{Si}_{3} \mathrm{~N}_{4}$ is deposited on a pre-defined silica platform followed by chemical mechanical polishing.

Our group has recently reported a novel fabrication technique using traditional subtractive processing and electron beam lithography (EBL) to directly pattern the as-deposited $\mathrm{Si}_{3} \mathrm{~N}_{4}$ film [4]. Compared with DUV stepper lithography, EBL has the ability to define small features down to $8 \mathrm{~nm}$. However, the writing field where stitching errors are avoided is only $1 \times 1 \mathrm{~mm}^{2}$, which makes it challenging to fabricate long waveguides. Wrapping the waveguide to fit within the writing field is possible but difficult to implement in practice because higher-order modes can be easily excited if the bends are not properly designed. In this work, we report high-Q $25 \mathrm{GHz}$ racetrack resonators. The cavity design minimizes the coupling to higher-order modes, allowing us to attain a smooth dispersion profile and realize single soliton microcombs. This achievement facilitates multiple applications of microcombs, particularly in the field of spectrally efficient coherent communications [5].

\section{Racetrack design and statistical analysis}

A finger-shape racetrack resonator, shown in Fig. 1(a), is adopted to achieve a cavity length $\sim 5700 \mu \mathrm{m}$ while keeping the pattern within the writing field of the EBL to avoid undesired stitching errors. A proper connection between straight segments needs to be designed, meaning that its tangent and curvature are continuous along the propagation of the light. We followed the design algorithm in [6] to minimize the variation of the curvature, which in turn diminishes the coupling to higher-order modes. The curvature is defined as $k(s)=a_{0}+a_{1} * s+a_{2} * s^{2}+a_{3} * s^{3}$, where $\mathrm{k}$ is the curvature and $\mathrm{s}$ the arc length. Boundary conditions based on start and end points are used to determine the polynomial coefficient. The devices are fabricated using the technique reported in [4]. The height and width of the microresonator waveguide are $740 \mathrm{~nm}$ x $2000 \mathrm{~nm}$.

We characterize the dispersion and Qs of fabricated devices using a method similar to [7]. The integrated dispersion (defined as $D_{\text {int }}=\omega_{\mu}-\omega_{0}-\mu D_{1}=D_{2} \mu^{2} / 2+D_{3} \mu^{3} / 6+\ldots$ where $\omega_{\mu}$ is the angular frequency of the $\mu$-th resonance relative to the reference resonance $\omega_{0}$, and $D_{1} / 2 \pi$ is the FSR) from the fundamental TE mode family is shown in Fig. 1(b). The retrieved values from the fitting are $\mathrm{D}_{1} / 2 \pi \sim 24.45 \mathrm{GHz}, \mathrm{D}_{2} / 2 \pi \sim 30.0 \mathrm{kHz}$. The converted $\beta_{2}$ is $-57.2 \pm 0.2 \mathrm{ps}^{2} / \mathrm{km}$ which is close to our simulation result $-50 \mathrm{ps}^{2} / \mathrm{km}$. Several avoided mode crossing points are observed in Fig. 1(b), but they are fairly weak and deviate less than $100 \mathrm{MHz}$ from the fitted third-order polynomial curve. This observation suggests that our designed connection curve minimized the coupling to higher order modes. The intrinsic Q of all the resonances within the spectrum from $1520 \mathrm{~nm}$ to $1620 \mathrm{~nm}$ are extracted, and the histogram of intrinsic Qs from 4 devices is shown in Fig.1 (c). The most probable intrinsic Q is 9 million. Few resonances with low intrinsic Qs are observed due to the avoided mode crossings. 
(a)

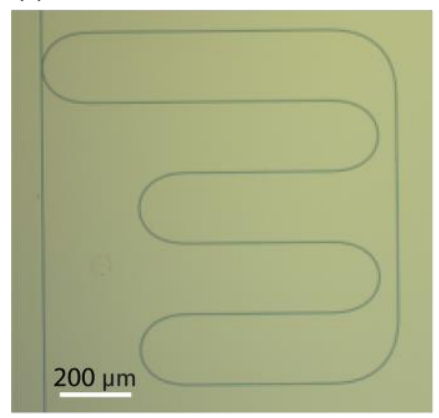

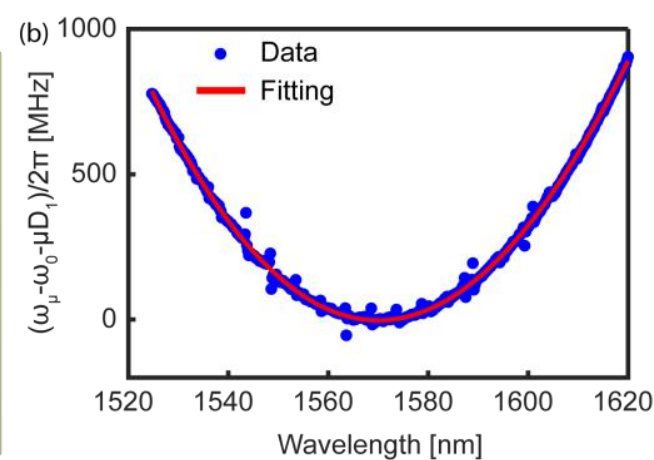

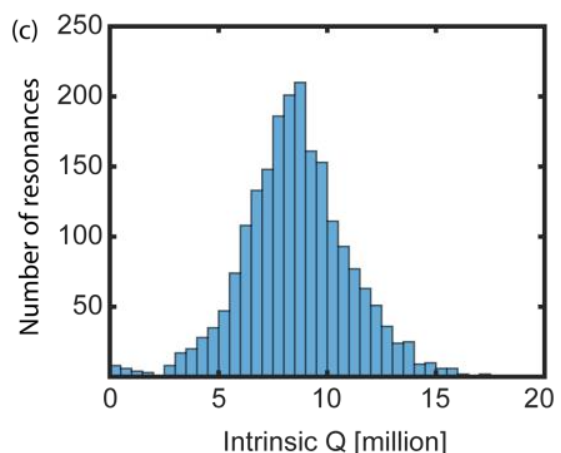

Fig. 1. (a) Microscope image of a fabricated $\mathrm{Si}_{3} \mathrm{~N}_{4}$ racetrack microresonator. (b) Integrated dispersion of the fundamental TE mode. (c) Histogram of the intrinsic Qs from 4 devices.

\section{Soliton microcomb generation}

In this section, we investigate soliton comb generation from high-Q $\mathrm{Si}_{3} \mathrm{~N}_{4}$ racetracks. We pump our device with an on-chip power $\sim 251 \mathrm{~mW}$, and the pump wavelength is fixed at $1553.7 \mathrm{~nm}$. Then we use a microheater to initialize the soliton microcomb. The converted power trace is shown in Fig. 2(a). The driving signal was stopped at the single soliton step, leading to the comb state whose spectrum is shown in Fig. 2(b). The continuous-wave pump line is suppressed in Fig. 2(b). The RF noise spectrum is shown in Fig. 2(c) confirming the low-noise state operation.

(a)

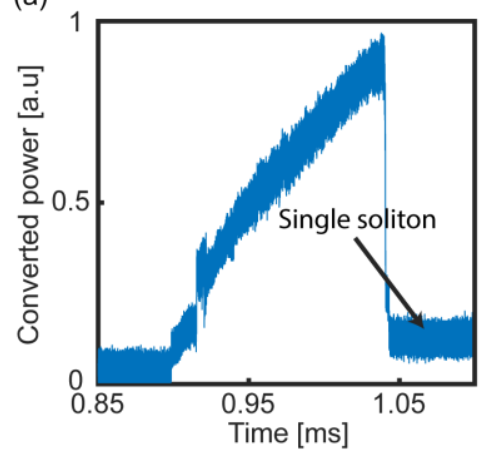

(b)

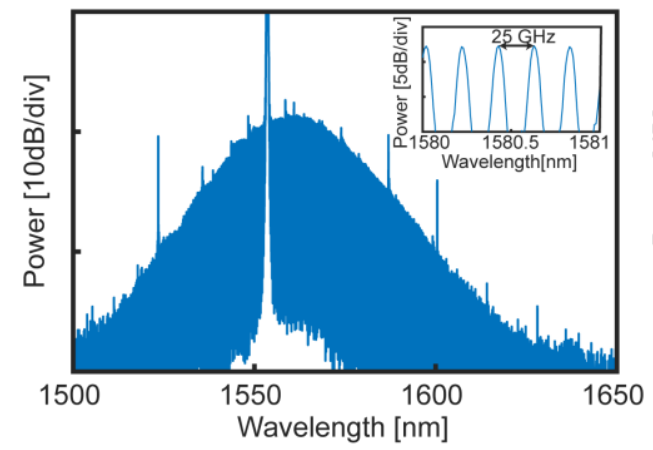

(c)

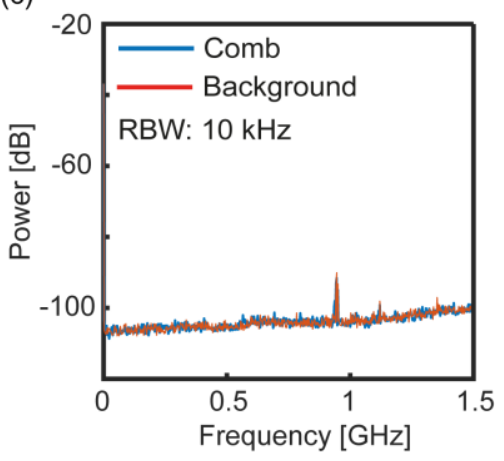

Fig. 2. (a) Converted power trace showing the single soliton step. (b) $25 \mathrm{GHz}$ single soliton comb generated with an on-chip power $\sim 251 \mathrm{~mW}$. Inset shows a zoom to the line spacing. (c) Corresponding RF spectrum.

In conclusion, we achieved a $25 \mathrm{GHz}$ single-soliton comb based on a $\mathrm{Si}_{3} \mathrm{~N}_{4}$ racetrack microresonator fabricated using a novel subtractive method. Coupling to higher-order modes is avoided thanks to a careful design of the connection curves. This method could be applied for longer cavities or even spiraled waveguides for diverse nonlinear optic applications.

We acknowledge support from the European Research Council (CoG 771410), the Swedish Research Council (2015-00535, 2016-03960, 2016-06077) and the H2020 Marie Curie Innovative Training Network Microcomb (812818).

\section{References}

1. T. J. Kippenberg et al., "Dissipative Kerr solitons in optical microresonators," Science 361, eaab8083 (2018).

2. A. R. Johnson et al., "Chip-based frequency combs with sub-100 GHz repetition rates," Opt. Lett. 37, 875 (2012)

3. J. Liu et al., "Nanophotonic soliton-based microwave synthesizers,", arXiv 1901.10372.

4. Z. Ye et al.,"High-Q $\mathrm{Si}_{3} \mathrm{~N}_{4}$ microresonators based on a subtractive processing for Kerr nonlinear optics,"Opt. Express 27, 35719-35727 (2019).

5. M. Mazur et al., "Enabling high spectral efficiency coherent superchannel transmission with soliton microcombs,", arXiv 1812.11046.

6. T. Chen et al., "A general design algorithm for low optical loss adiabatic connections in waveguides," Opt. Express 20, 22819-22829 (2012).

7. S. W. Huang et al., "Mode-locked ultrashort pulse generation from on-chip normal dispersion...," Phys. Rev. Lett. 114, 053901 (2015). 